OPEN Antisense Oligonucleotides Targeting

SUBJECT AREAS:

ANTISENSE

OLIGONUCLEOTIDE

THERAPY

PARASITIC INFECTION

PARASITE PHYSIOLOGY

CALCIUM SIGNALLING

Received

18 March 2013

Accepted

6 February 2014

Published

28 February 2014

Correspondence and requests for materials should be addressed to T.N. (tnara@juntendo. ac.jp) or K.M.

(mikosiba@brain.

riken.jp)

\section{Parasite Inositol 1,4,5-Trisphosphate Receptor Inhibits Mammalian Host Cell Invasion by Trypanosoma cruzi}

Muneaki Hashimoto' ', Takeshi Nara', Hiroko Hirawake', Jorge Morales' , Masahiro Enomoto ${ }^{2}$ \& Katsuhiko Mikoshiba ${ }^{3,4}$

'Department of Molecular and Cellular Parasitology, Juntendo University School of Medicine, 2-1-1 Hongo, Bunkyo-ku, Tokyo $113-$ 8421 , Japan, ${ }^{2}$ Division of Signaling Biology, Ontario Cancer Institute, Toronto, ON, Canada M5G 1L7, ${ }^{3}$ Laboratory for Developmental Neurobiology, RIKEN Brain Science Institute, Saitama 351-0198, Japan, ${ }^{4}$ Calcium Oscillation Project, International Cooperative Research Project and Solution-Oriented Research for Science and Technology, Japan Science and Technology Agency, Kawaguchi, Saitama 332-0012, Japan.

Chagas disease is caused by an intracellular parasitic protist, Trypanosoma cruzi. As there are no highly effective drugs against this agent that also demonstrate low toxicity, there is an urgent need for development of new drugs to treat Chagas disease. We have previously demonstrated that the parasite inositol 1,4,5-trisphosphate receptor $\left(\mathrm{TcIP}_{3} \mathrm{R}\right)$ is crucial for invasion of the mammalian host cell by T. cruzi. Here, we report that $\operatorname{TcIP}_{3} \mathrm{R}$ is a short-lived protein and that its expression is significantly suppressed in trypomastigotes. Treatment of trypomastigotes, an infective stage of $T$. cruzi, with antisense oligonucleotides specific to $T c I P_{3} R$ deceased $T c I P_{3} R$ protein levels and impaired trypomastigote invasion of host cells. Due to the resulting instability and very low expression level of $\operatorname{TcIP}_{3} R$ in trypomastigotes indicates that $\mathrm{TcIP}_{3} \mathrm{R}$ is a promising target for antisense therapy in Chagas disease.

T he parasitic protist, Trypanosoma cruzi, is an etiologic agent of Chagas disease ${ }^{1}$. Human infection is initiated by invasion of infective, metacyclic trypomastigotes in the urine of blood-sucking reduviid bugs ${ }^{2}$. After invasion, the parasite transforms into amastigotes inside the host cells and begins to proliferate. Parasite proliferation is often accompanied by destruction of the vital tissues, such as heart muscle. Chemotherapy of Chagas disease relies exclusively on two drugs, benznidazole and nifurtimox, but their effects are limited and often evoke severe side effects. Therefore, development of new therapeutic measures are urgently needed.

$\mathrm{Ca}^{2+}$ serves as an second messenger of cellular signaling and its concentration is strictly maintained in the cytosol at $0.1 \mu \mathrm{M}$ order ${ }^{3}$. Transient increase of intracellular $\mathrm{Ca}^{2+}$ concentration plays a crucial role for its functions and is mediated in response to both external and internal stimuli. D-myo-inositol 1,4,5-trisphosphate $\left(\mathrm{IP}_{3}\right)$ is a second messenger generated by phosphoinositide phospholipase C (PI-PLC) upon external stimuli via cell-surface receptors ${ }^{4}$ and provokes activation of its receptor, $\mathrm{IP}_{3} \mathrm{R} . \mathrm{IP}_{3} \mathrm{R}$ is a $\mathrm{Ca}^{2+}$ channel located on the endoplasmic reticulum (ER) and is activated by the binding of $\mathrm{IP}_{3}$, which initiates $\mathrm{Ca}^{2+}$ release from $\mathrm{ER}$ as a $\mathrm{Ca}^{2+}$ pool $^{5}$.

Recently, we reported that a homologue of $\mathrm{IP}_{3} \mathrm{R}$ in $T$. cruzi $\left(\mathrm{TcIP}_{3} \mathrm{R}\right)$ is an essential protein and participates in the growth and transformation of the parasite and its ability to infect the host cell. Furthermore, we demonstrated that $\mathrm{TcIP}_{3} \mathrm{R}$ is a determinant of the virulence of the parasite in vivo ${ }^{6}$. Combined with the fact that the primary structure of $\mathrm{TcIP}_{3} \mathrm{R}$ has low similarity to that of human $\mathrm{IP}_{3} \mathrm{Rs}, \mathrm{TcIP}_{3} \mathrm{R}$ is a promising drug target for Chagas disease.

Antisense oligonucleotides are a new generation of therapeutic agents that work by silencing the genes responsible for the diseases. One example is fomivirsen (marketed as Vitravene ${ }^{\circledR}$ ), which has been approved by the US Food and Drug Administration (FDA) in 1998 for the treatment of cytomegalovirus retinitis ${ }^{7}$. In parasitic infections, use of antisense oligonucleotides to inhibit specific mRNA synthesis and translation may represent a good chemotherapeutic strategy ${ }^{8}$. There are several reports showing that treatment of T. cruzi in vitro with 
antisense oligonucleotides decreased expression levels of the target proteins $^{9-11}$. Thus, an antisense strategy against pivotal proteins of $T$. cruzi holds promise for a new treatment for Chagas disease.

In the present study, we examined whether $\operatorname{TcIP}_{3} \mathrm{R}$ is a potential target for antisense oligonucleotide treatment against $T$. cruzi by phenotypic analysis of trypomastigotes in an in vitro culture system. We show considerably reduced levels of parasite invasion of host cells, implying that antisense oligonucleotide chemotherapy against $\mathrm{TcIP}_{3} \mathrm{R}$ may be a viable approach to treatment in Chagas disease.

\section{Results}

$\mathrm{TcIP}_{3} \mathbf{R}$ is a short-lived protein in epimastigotes. Since antisense oligonucleotides specifically interfere with both mRNA stability and its translation into protein, short-lived proteins are desirable targets to ensure effective, functional knock-down by antisense oligonucleotides. In order to establish whether $\mathrm{TcIP}_{3} \mathrm{R}$ is suitable as a target for antisense strategy, we treated T. cruzi epimastigotes with cycloheximide (CHX), an authentic inhibitor of protein synthesis, for $0.5-10 \mathrm{~h}$, and monitored degradation of $\mathrm{TcIP}_{3} \mathrm{R}$ by western blot analysis (Fig. 1A). Expression levels of $\mathrm{TcIP}_{3} \mathrm{R}$ declined after $\mathrm{CHX}$ treatment, whereas it was difficult to estimate its half-life, due exclusively to its low levels of expression.

We have recently established T. cruzi that overexpress recombinant $\mathrm{TcIP}_{3} \mathrm{R}$ fused to enhanced green fluorescent protein (EGFP) at its $\mathrm{N}$-terminal (EGFP-TcIP ${ }_{3} \mathrm{R}$ ), which is physiologically functional in the parasite ${ }^{6}$. We examined the inhibitory effect of CHX treatment on expression of EGFP-TcIP ${ }_{3} \mathrm{R}$ to ascertain whether $\mathrm{TcIP}_{3} \mathrm{R}$ domainspecific protein degradation occurs. Western blots showed that the protein signals of EGFP-TcIP ${ }_{3} \mathrm{R}$ decreased rapidly and became undetectable by $8 \mathrm{~h}$ after CHX treatment, whereas the band for EGFP remained almost intact (Fig. 1B). These results clearly indicated that the degradation of EGFP-TcIP ${ }_{3} \mathrm{R}$ is specific to the $\mathrm{TcIP}_{3} \mathrm{R}$ domain. The half-life of EGFP-TcIP ${ }_{3} \mathrm{R}$ was estimated to be about $3 \mathrm{~h}$, while the half-life of mammalian $\mathrm{IP}_{3} \mathrm{Rs}$ in unstimulated cultured cells is 10-12 $\mathrm{h}^{12}$, suggesting that $\operatorname{TcIP}_{3} \mathrm{R}$ is more unstable than mammalian $\mathrm{IP}_{3} \mathrm{Rs}$. We concluded that $\mathrm{TcIP}_{3} \mathrm{R}$ is a short-lived protein at least in epimastigotes, and possibly other forms of T. cruzi.

Protein level of $\operatorname{TcIP}_{3} R$ is very low in trypomastigotes. We have recently shown that transcription of $T c I P_{3} R$ mRNA occurs throughout the parasite life cycle, but that its transcription level was much lower in trypomastigotes than in epimastigotes ${ }^{6}$. In the present study, the protein levels of $\mathrm{TcIP}_{3} \mathrm{R}$ were examined by western blotting using an anti- $\mathrm{TcIP}_{3} \mathrm{R}$ monoclonal antibody, and were compared between epimastigotes and trypomastigotes. $\mathrm{TcIP}_{3} \mathrm{R}$ was detected in epimastigotes, but was undetectable in trypomastigotes, while the levels of $\beta$-tubulin, a control protein, were consistent between the 2 parasite forms (Fig. 1C). These results indicated that the protein level of $\mathrm{TcIP}_{3} \mathrm{R}$ is very low in trypomastigotes.

Because the native $\mathrm{TcIP}_{3} \mathrm{R}$ protein is undetectable in trypomastigotes, we tested whether EGFP-TcIP ${ }_{3} \mathrm{R}$ was detectable in trypomastigotes of the transgenic T. cruzi. We could detect EGFP-TcIP ${ }_{3} \mathrm{R}$ by western blots using an EGFP-specific antibody, confirming that these EGFP-TcIP ${ }_{3} \mathrm{R}$-expressing T. cruzi were suitable for further analysis (Fig. 2). Notably, expression levels of EGFP-TcIP ${ }_{3} \mathrm{R}$ in trypomastigotes (Fig. 2A, untreated) was very low and was reduced to less than $10 \%$ of that in epimastigotes (Fig. 2, epimastigotes), consistent with the results found in the wild-type parasite.

Expression of $\mathrm{TcIP}_{3} \mathrm{R}$ is blocked by the antisense oligonucleotide treatment. It has been reported that antisense oligonucleotides can be incorporated into non-dividing, infective trypomastigotes by coincubation in medium, without specific treatment, leading to an effective knock-down of target protein expression ${ }^{10}$. Given this, and the fact that trypomastigotes are the exclusive invasive form in
A
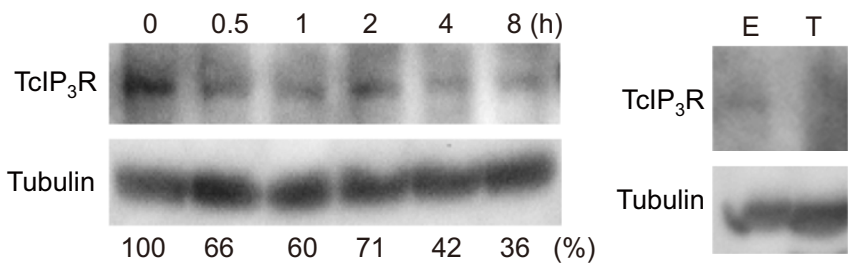

B
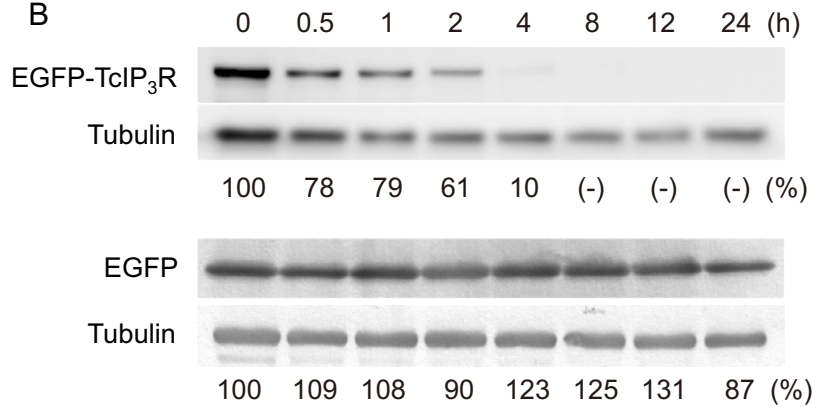

Figure $1 \mid$ Expression levels of $\mathrm{TcIP}_{3} \mathrm{R}$ protein in Trypanosoma cruzi. (A) Life-span of $\mathrm{TcIP}_{3} \mathrm{R}$ in epimastigotes. T. cruzi epimastigotes were incubated with $200 \mu \mathrm{g} / \mathrm{mL}$ cycloheximide (CHX) for the indicated time, and expression of $\mathrm{TcIP}_{3} \mathrm{R}$ was analyzed by western blotting using anti$\mathrm{TcIP}_{3} \mathrm{R}$ antibody. The expression levels of $\mathrm{TcIP}_{3} \mathrm{R}$ were normalized to the levels of tubulin and the relative ratio (\%) was indicated. (B) Life-span of recombinant $\mathrm{TcIP}_{3} \mathrm{R}$ in epimastigotes. Epimastigotes overexpressing EGFP-TcIP ${ }_{3} \mathrm{R}$ fusion protein were incubated with $200 \mu \mathrm{g} / \mathrm{mL}$ CHX for the indicated time, and the expression of EGFP-TcIP ${ }_{3} \mathrm{R}$ was analyzed by western blotting using anti- $\mathrm{TcIP}_{3} \mathrm{R}$ antibody. Epimastigotes expressing EGFP was used as control, and western blots were probed with anti-EGFP antibody. Tubulin was used as loading control. Parallel images were processed from the same gel. The levels of EGFP-TcIP 3 R were normalized to tubulin expression level using a freeware, ImageJ Ver. 1.47 and the relative ratio (\%) was indicated. (C) Suppressed expression of $\mathrm{TcIP}_{3} \mathrm{R}$ in trypomastigotes. Cell extracts from epimastigotes (E) or trypomastigotes (T) were resolved by SDS-PAGE, and western blots were probed with anti$\mathrm{TcIP}_{3} \mathrm{R}$ antibody or anti-tubulin antibody as an internal control. Note that $\mathrm{TcIP}_{3} \mathrm{R}$ was undetectable in trypomastigotes. Full-length blots of panels $\mathrm{A}$, B, and C are presented in Supplementary Figure S1, S2, and S3, respectively.

non-phagocytic cells, we selected trypomastigotes as the target stage of the parasite for antisense treatment.

Because of the difficulty to determine the stability of native $\mathrm{TcIP}_{3} \mathrm{R}$, we addressed whether treatment with antisense oligonucleotides inhibits expression of EGFP-TcIP ${ }_{3} \mathrm{R}$ in trypomastigotes (Fig. 2). Expression levels of EGFP-TcIP ${ }_{3} \mathrm{R}$ in trypomastigotes treated with the antisense oligonucleotide (Antisense 5995) was significantly reduced to $54 \%$ (vs. untreated; $p=0.003)$ and $41 \%(p=0.0006)$ after $4 \mathrm{~h}$ and $8 \mathrm{~h}$ treatment, respectively (Fig. 2B). Although the stability of $\mathrm{TcIP}_{3} \mathrm{R}$ in trypomastigotes is unclear, reduction of the protein levels of EGFP-TcIP ${ }_{3} \mathrm{R}$ is only attributable to the degradation of premade proteins under the conditions that protein synthesis is suppressed by antisense oligonucleotide treatment, as well as by $\mathrm{CHX}$ treatment (see also Fig. 1). This is also supported by the fact that the reduction of EGFP-TcIP ${ }_{3} \mathrm{R}$ levels is time-dependent. Therefore, it is likely that EGFP-TcIP ${ }_{3}$ p protein, and possibly native $\mathrm{TcIP}_{3} \mathrm{R}$, are a short-lived protein in trypomastigotes.

Treatment with the complementary sense oligonucleotide (Sense 5995S) also showed 54\% reduction of EGFP-TcIP ${ }_{3}$ R expression after $4 \mathrm{~h}$ treatment (vs. untreated; $p=0.017$ ), whereas the effect was rather limited. This was probably due to association of the sense 

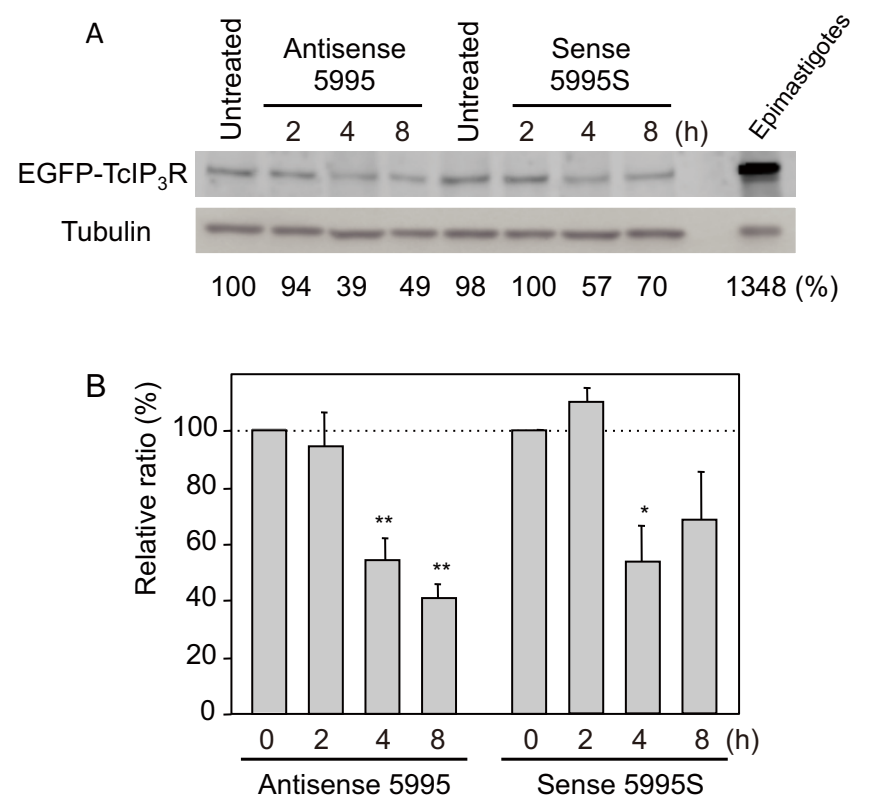

Figure $2 \mid$ Phosphorothioate antisense $\mathrm{TcIP}_{3} \mathrm{R}$ oligonucleotide inhibits EGFP-TcIP ${ }_{3} \mathrm{R}$ expression in trypomastigotes. (A) Western blots of trypomastigotes overexpressing EGFP-TcIP ${ }_{3} \mathrm{R}$, treated for $8 \mathrm{~h}$ with $40 \mu \mathrm{M}$ of either Antisense 5995 or the complementary Sense 5995S

phosphorothioate oligonucleotide, were probed with anti-EGFP antibody or an anti-tubulin antibody as internal control. The lysate of EGFP-

$\mathrm{TcIP}_{3} \mathrm{R}$-expressing epimastigotes without treatment was loaded as expression control. Parallel images were processed from the same gel. The expression levels of $\mathrm{TcIP}_{3} \mathrm{R}$ were normalized to the levels of tubulin and the relative ratio (\%) is indicated. Representative data of three independent experiments are shown. Full-length blots are presented in Supplementary Figure S4. (B) Decrease of the expression levels of EGFP-TcIP ${ }_{3} \mathrm{R}$ in trypomastigotes treated with Antisense 5995 or Sense 5995S. Intensity of the bands was measured densitometrically using a freeware, ImageJ version 1.47. Bars indicate the mean \pm S.E. $(\mathrm{n}=3)$. Statistical differences $\left({ }^{*} p<\right.$ $\left.0.05,{ }^{* *} p<0.01\right)$ are given as a comparison between untreated $(0)$ and treated $(2,4$, and 8 hours) groups.

oligonucleotide with the antisense DNA strand, which may interfere with transcription and lead to the inhibition of transcription.

Infectivity of trypomastigotes is decreased by treatment with antisense oligonucleotides targeted against $\operatorname{TcIP}_{3} R$ mRNA. To establish whether treatment with oligonucleotides led to impairment of trypomastigote invasion, we compared the inhibitory effects between Antisense 5995 and Sense 5995S on trypomastigotes. By $8 \mathrm{~h}$ after treatment of trypomastigotes with Antisense 5995 , invasion by parasites was significantly inhibited (vs. control; $p<0.001$, vs. Sense 5995S; $p<0.05$; Fig. 3A). These results strongly suggested that loss of infectivity by trypomastigotes is mediated by treatment with antisense oligonucleotides that result in knock-down of $\mathrm{TcIP}_{3} R$.

We further tested the inhibitory effects of additional 3 antisense oligonucleotides (Antisense 5531, 1777, and 8646) on invasion of HeLa cells by trypomastigotes. Treatment of trypomastigotes with each of these antisense oligonucleotides resulted in impaired invasion of HeLa cells by the parasite (Fig. 3B). These results indicated that suppression of expression of $\mathrm{TcIP}_{3} R$ is dependent on the antisense oligonucleotide sequence, and can result in impaired trypomastigote infectivity.

\section{Discussion}

Chemotherapy of Chagas disease currently relies essentially on 2 old compounds, benznidazole and nifurtimox, both of which elicit

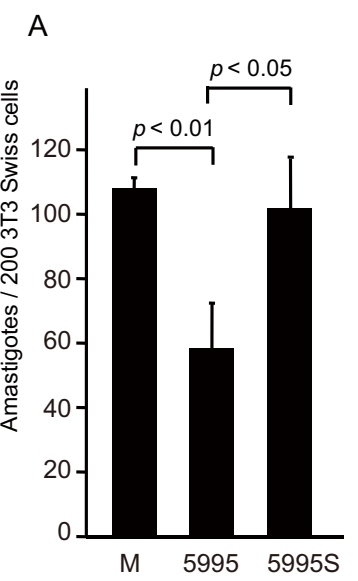

B

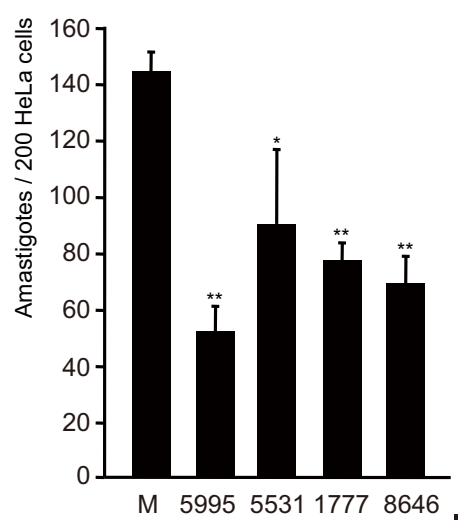

Figure 3 Inhibitory effect of antisense $\mathrm{TcIP}_{3} \mathrm{R}$ oligonucleotides on trypomastigote cell invasion. (A) Trypomastigotes $\left(2 \times 10^{5}\right)$ were treated for $8 \mathrm{~h}$ with $40 \mu \mathrm{M}$ of either Antisense 5995 or its complementary Sense $5995 \mathrm{~S}$ and were then incubated with $2 \times 10^{4} 3 \mathrm{~T} 3$-Swiss albino cells for $12 \mathrm{~h}$ at $37^{\circ} \mathrm{C}$ at a multiplicity of infection (MOI) of 10 . (B) Trypomastigotes (4 $\times 10^{5}$ ) were treated with $40 \mu \mathrm{M}$ of phosphorothioate oligonucleotides (5995, 5531, 1777, and 8646, see Materials and Methods) for $8 \mathrm{~h}$ and were then incubated with $4 \times 10^{4} \mathrm{HeLa}$ cells for $12 \mathrm{~h}$ at $37^{\circ} \mathrm{C}(10 \mathrm{MOI})$. For calculation of trypomastigote infectivity, the number of intracellular parasites in a total of 200 cells was counted after Giemsa staining. Data shown are the mean \pm S.D. of 3 independent experiments. Statistical differences $\left({ }^{*} p<0.05,{ }^{* *} p<0.01\right)$ are given as a comparison between the mock $(M)$ and experimental groups $(n=3)$.

harmful side effects. Therefore, development of novel drugs for treatment of this disease is crucial. New therapeutic measures against infectious diseases include antisense oligonucleotides, which aim to knock down an essential component in the responsible pathogens. We recently reported that when the expression level of $\mathrm{TcIP}_{3} \mathrm{R}$ decreased to only two-thirds of that of the wild-type, invasion of $T$. cruzi trypomastigotes was significantly impaired, indicating that $\operatorname{TcIP}_{3} \mathrm{R}$ is a potential therapeutic target ${ }^{6}$. In light of the fact that a canonical RNA interference pathway is absent in T. $c r u z i^{13}$, we evaluated knock-down of $\mathrm{TcIP}_{3} \mathrm{R}$ using antisense oligonucleotides as a practical measure.

We found that $\operatorname{TcIP}_{3} \mathrm{R}$ is a short-lived protein at least in epimastigotes. Consistently, the recombinant EGFP-TcIP ${ }_{3} \mathrm{R}$ is also a shortlived protein in epimastigotes, due to $\mathrm{TcIP}_{3} \mathrm{R}$ domain-specific protein degradation. In trypomastigotes, we found significant reduction of the protein levels of EGFP-TcIP ${ }_{3} \mathrm{R}$ by antisense oligonucleotide treatment in a time-dependent manner, suggesting that EGFP$\mathrm{TcIP}_{3} \mathrm{R}$ is rapidly degraded in trypomastigotes (54\% reduction after $4 \mathrm{~h}$ treatment, Fig. 2). Therefore, it is likely that $\mathrm{TcIP}_{3} \mathrm{R}$ is a shortlived protein in both epimastigotes and trypomastigotes. Notably, mammalian $\mathrm{IP}_{3}$ Rs are post-translationally regulated by degradation via the ubiquitin-proteasome pathway ${ }^{14}$. Thus, these findings have important implications for development of antisense therapy against $\mathrm{TcIP}_{3} \mathrm{R}$.

Upon attachment to the host cell, $\mathrm{IP}_{3} \mathrm{R}$-dependent $\mathrm{Ca}^{2+}$ release is evident in trypomastigotes ${ }^{6}$, indicating the presence and a physiological role of $\mathrm{TcIP}_{3} \mathrm{R}$ in trypomastigotes. Notably, mRNA and protein levels of $\mathrm{TcIP}_{3} \mathrm{R}$ were found to be very low in trypomastigotes, when compared with those in epimastigotes. However, it was difficult to detect and even quantify the turnover of $\mathrm{TcIP}_{3} \mathrm{R}$ protein in trypomastigotes by western blots, most likely due to the very low transcription/expression levels of $\mathrm{TcIP}_{3} \mathrm{R}$. In contrast, EGFP-TcIP $\mathrm{R}$ was detectable in trypomastigotes of the recombinant parasites and, importantly, its expression level in trypomastigotes was less than $10 \%$ of that in epimastigotes. 
In the present study, EGFP-TcIP ${ }_{3} \mathrm{R}$ was expressed using pTREX expression vector via the ribosomal RNA promoter that facilitates constitutive, high-level transcription ${ }^{15}$. Therefore, it is likely that $\mathrm{TcIP}_{3} \mathrm{R}$-specific protein degradation is more active in trypomastigotes than in epimastigotes, while we cannot exclude the possibility that the transcription level of EGFP-TcIP ${ }_{3} \mathrm{R}$, as well as its protein level, in trypomastigotes is very low.

Reduction of infectivity of trypomastigotes after treatment with $\mathrm{TcIP}_{3} \mathrm{R}$-specific antisense oligonucleotides strongly suggested that the suppression of transcription of this gene led to reduced levels of $\mathrm{TcIP}_{3} \mathrm{R}$ protein, which occurred in conjunction with rapid turnover at the protein level in trypomastigotes. Thus, expression levels of $\mathrm{TcIP}_{3} \mathrm{R}$ is tightly regulated in trypomastigotes at both transcription and protein levels.

Low expression levels of $\mathrm{TcIP}_{3} \mathrm{R}$ in trypomastigotes, as well as its rapid turnover, are advantageous for further development of antisense therapy against $\mathrm{TcIP}_{3} \mathrm{R}$. Firstly, trypomastigotes are the only invasive stage of T. cruzi, and are responsible for the virulence of the parasite. Therefore, efficient targeting of the relatively small copy number of $T c I P_{3} R$ transcripts by introduction of antisense oligonucleotides followed by suppression of infectivity of trypomastigotes should be possible. Secondly, trypomastigotes represent the nondividing stage, so that the intracellular concentration of antisense oligonucleotides can be stabilized. It is worth noting that when antisense nucleotides are incorporated in the dividing stage of the parasites (e.g. epimastigotes or amastigotes), the concentration of the oligonucleotide in the parasites may become diluted as the parasite replicates. Thirdly, antisense oligonucleotides can be incorporated into trypomastigotes without artificial treatment ${ }^{10}$, which facilitates the strategy.

In terms of Chagas disease, the antisense approach is suitable particularly in the acute phase, in which the blood-circulating trypomastigotes predominate, whereas they are often undetectable in other phases, such as the indeterminate and chronic stages of infection. In addition, it is important to know whether this therapeutic approach is effective not only in trypomastigotes, but also in amastigotes. We are now planning to investigate this using an in vitro infection system and an experimental animal model. In conjunction with the fact that $\mathrm{TcIP}_{3} \mathrm{R}$ shares far less similarity with mammalian $\mathrm{IP}_{3} \mathrm{Rs}^{6}, \mathrm{TcIP}_{3} \mathrm{R}$ holds great promise as a target for antisense treatment with reduced side-effects.

It has been reported that PI-PLC of T. cruzi (TcPI-PLC) is essential for the parasite, and TcPI-PLC has been shown to be related to trypomastigote-to-amastigote differentiation by experiments using antisense oligonucleotides ${ }^{9}$. Since PI-PLC synthesizes $\mathrm{IP}_{3}$, followed by stimulation of $\mathrm{TcIP}_{3} \mathrm{R}$ by the generated $\mathrm{IP}_{3}$, the antisense oligonucleotides against TcPI-PLC may also inhibit trypomastigote invasion of the host cell. Interestingly, the mRNA levels of TcPI-PLC are lower in trypomastigotes than in epimastigotes and amastigotes ${ }^{16}$, similar to that of $\mathrm{TcIP}_{3} \mathrm{R}$. This suggests the particular physiological importance of $\mathrm{IP}_{3}$-mediated $\mathrm{Ca}^{2+}$ signaling during the mammalian stage of infection.

We could significantly inhibit parasite invasion of the host cells by antisense oligonucleotide treatment, but this effect was incomplete. This is probably due to inefficient knock-down by the phosphorothioate oligonucleotides used in the present study. It is important to note that T. cruzi with $\mathrm{TcIP}_{3} \mathrm{R}$-knock-down (in which 1 of 2 gene loci was disrupted), rather than null-mutants, became thoroughly avirulent in the experimental murine model, whereas in trypomastigotes with this knock-down showed only a $40 \%$ reduction in infectivity of the host cell ${ }^{6}$. In the present study, antisense treatment showed comparable levels of reduction in infectivity. Therefore, it is possible that in vivo antisense treatment can be effective for preventing development of the disease.

Knock-down efficacy may be improved by using antisense oligonucleotides with $2^{\prime}-O, 4^{\prime}-C$-Ethylene-bridged nucleic acid species
(ENAs $)^{17}$. ENAs have higher binding affinity for the complementary RNA strand and are more resistant to nucleases than are phosphorothioate nucleic acids. Therefore, ENA antisense oligonucleotides are more favorable from a therapeutic viewpoint. Further analysis using ENA-based antisense oligonucleotides and an experimental animal model is necessary to optimize the conditions for this therapeutic strategy against Chagas disease.

\section{Methods}

Parasite and host cells. Epimastigotes of the T. cruzi Tulahuen strain were cultured as described $^{18}$. Metacyclic development was induced as previously described ${ }^{19}$. Mammalian stages of the parasites were maintained in in vitro culture using 3T3Swiss albino cells (Health Science Research Bank, Tokyo, Japan) and tissue culturegrown trypomastigotes were collected from the culture supernatants by centrifugation, essentially as previously described ${ }^{20}$. For in vitro experimental infection, 3T3-Swiss albino cells and human-derived HeLa cells were used.

Antibodies and reagents. The anti-TcIP3R monoclonal antibody was prepared as described previously ${ }^{6}$. The anti-EGFP and anti-tubulin antibodies were purchased from Molecular Probes, Inc. (Eugene, OR) and Thermo Fisher Scientific, Inc. (Rockford, IL), respectively. Cycloheximide was purchased from Wako Pure Chemicals Industries, Ltd. (Osaka, Japan). Quick-CBB PLUS (Wako) was used for CBB staining. Western blotting was performed as described ${ }^{21}$.

Oligonucleotides. The following phosphorothioate oligonucleotides were designed and purchased from Integrated DNA Technologies, Inc. (Diego, CA). Antisense 1777, 5531,5995 and 8649 correspond to the complementary sequence of $T c I P_{3} R$ gene, $5^{\prime}$ TTCCAAGCCTCCACCATCCC-3', 5' -TCTCTTCCCAGCCACCACCT-3', , 5' GTCCTCCCTTTCCGTGCTGT-3', and 5' -TCCTCCTCCCTTCCGCCATT-3', respectively. Sense Oligonucleotide, 5995S (5' -ACAGCACGGAAAGGGAGGAC$\left.3^{\prime}\right)$, is complementary to Antisense 5995.

Statistical analysis. Statistical analysis between the groups was performed using oneway ANOVA and Fisher's PLSD post hoc test.

1. Rassi, A., Jr., Rassi, A. \& Marin-Neto, J. A. Chagas disease. Lancet. 375, 1388-1402 (2010).

2. Brener, Z. Biology of Trypanosoma cruzi. Annu Rev Microbiol. 27, 347-382 (1973).

3. Bootman, M. D., Lipp, P. \& Berridge, M. J. The organisation and functions of local $\mathrm{Ca}^{2+}$ signals. J Cell Sci. 114, 2213-2222 (2001).

4. Berridge, M. J. Inositol trisphosphate and calcium signalling. Nature. 361, 315-325 (1993).

5. Berridge, M. J., Bootman, M. D. \& Roderick, H. L. Calcium signalling: dynamics, homeostasis and remodelling. Nat Rev Mol Cell Biol. 4, 517-529 (2003).

6. Hashimoto, M. et al. Inositol 1,4,5-trisphosphate receptor regulates replication, differentiation, infectivity and virulence of the parasitic protist Trypanosoma cruzi. Mol Microbiol. (2013).

7. Razonable, R. R. Antiviral drugs for viruses other than human immunodeficiency virus. Mayo Clin Proc. 86, 1009-1026 (2011).

8. Barker, R. H., Jr., Metelev, V., Rapaport, E. \& Zamecnik, P. Inhibition of Plasmodium falciparum malaria using antisense oligodeoxynucleotides. Proc Natl Acad Sci U S A. 93, 514-518 (1996).

9. Okura, M. et al. A lipid-modified phosphoinositide-specific phospholipase C (TCPI-PLC) is involved in differentiation of trypomastigotes to amastigotes of Trypanosoma cruzi. J Biol Chem. 280, 16235-16243 (2005).

10. Málaga, S. \& Yoshida, N. Targeted reduction in expression of Trypanosoma cruzi surface glycoprotein gp90 increases parasite infectivity. Infect Immun. 69, 353-359 (2001).

11. Araya, J. E. et al. Calcineurin B of the human protozoan parasite Trypanosoma cruzi is involved in cell invasion. Microbes Infect. 10, 892-900 (2008).

12. Wojcikiewicz, R. J. H. Inositol 1,4,5-trisphosphate receptor degradation pathways. WIREs Membr Transp Signal. 1, 126-135 (2012).

13. Ullu, E., Tschudi, C. \& Chakraborty, T. RNA interference in protozoan parasites. Cell Microbiol. 6, 509-519 (2004).

14. Lu, J. P., Wang, Y., Sliter, D. A., Pearce, M. M. \& Wojcikiewicz, R. J. RNF170 protein, an endoplasmic reticulum membrane ubiquitin ligase, mediates inositol 1,4,5-trisphosphate receptor ubiquitination and degradation. J Biol Chem. 286, 24426-24433 (2011).

15. Lorenzi, H. A., Vazquez, M. P. \& Levin, M. J. Integration of expression vectors into the ribosomal locus of Trypanosoma cruzi. Gene. 310, 91-99 (2003).

16. Furuya, T., Kashuba, C., Docampo, R. \& Moreno, S. N. A novel phosphatidylinositol-phospholipase C of Trypanosoma cruzi that is lipid modified and activated during trypomastigote to amastigote differentiation. J Biol Chem. 275, 6428-6438 (2000).

17. Takagi, M. et al. Enhancement of the inhibitory activity of oatp antisense oligonucleotides by incorporation of $2^{\prime}$ - $O, 4^{\prime}$ - $C$-ethylene-bridged nucleic acids (ENA) without a loss of subtype selectivity. Biochemistry. 43, 4501-4510 (2004). 
18. Iizumi, K. et al. Molecular cloning and characterization of ouabain-insensitive $\mathrm{Na}(+)$-ATPase in the parasitic protist, Trypanosoma cruzi. Biochim Biophys Acta. 1758, 738-746 (2006).

19. Gluenz, E., Taylor, M. C. \& Kelly, J. M. The Trypanosoma cruzi metacyclic-specific protein Met-III associates with the nucleolus and contains independent amino and carboxyl terminal targeting elements. Int J Parasitol. 37, 617-625 (2007).

20. Nakajima-Shimada, J., Hirota, Y. \& Aoki, T. Inhibition of Trypanosoma cruzi growth in mammalian cells by purine and pyrimidine analogs. Antimicrob Agents Chemother. 40, 2455-2458 (1996).

21. Murata, E., Hashimoto, M. \& Aoki, T. Interaction between cFLIP and Itch, a ubiquitin ligase, is obstructed in Trypanosoma cruzi-infected human cells. Microbiol Immunol. 52, 539-543 (2008).

\section{Acknowledgments}

This work was supported in part by grants-in-aid for scientific research Nos. 24390102 (to T. Nara); and 25221002 (to K. Mikoshiba) and by the Foundation of Strategic Research Projects in Private Universities (S1201013; to T. Nara) from the Ministry of Education, Culture, Sport, Science, and Technology, Japan (MEXT).

\section{Author contributions}

M.H., T.N. and K.M. designed the study. M.H., H.H. and T.N. did the experiments. M.H. and T.N. wrote the manuscript. M.H., M.E., J.M. and K.M. interpreted the data. All authors reviewed the manuscript.

\section{Additional information}

Supplementary information accompanies this paper at http://www.nature.com/ scientificreports

Competing financial interests: The authors declare no competing financial interests.

How to cite this article: Hashimoto, M. et al. Antisense Oligonucleotides Targeting Parasite Inositol 1,4,5-Trisphosphate Receptor Inhibits Mammalian Host Cell Invasion by Trypanosoma cruzi. Sci. Rep. 4, 4231; DOI:10.1038/srep04231 (2014).

(c) (1) $\Theta($ This work is licensed under a Creative Commons Attribution-

By NC No NonCommercial-NoDerivs 3.0 Unported license. To view a copy of this license, visit http://creativecommons.org/licenses/by-nc-nd/3.0 